This document is the accepted manuscript version of the following article:

Colvi11, E., Krieger, M., Bosshard, P., Steinacher, P., Rohrer Schnidrig, B. A.,

Parke1, T., ... Fattori, G. (2020). Anthropomorphic phantom for deformable lung and liver CT and MR imaging for radiotherapy. Physics in Medicine and Biology, 65(7), 07NT02 (10 pp.). https://doi.org/10.1088/1361-6560/ab7508

\title{
Anthropomorphic phantom for deformable lung and liver CT and MR imaging for radiotherapy
}

Emma Colvill' ${ }^{1}$, Miriam Krieger ${ }^{1,2}$, Patrick Bosshard ${ }^{2}$, Patrice Steinacher ${ }^{1}$, Benno Andreas Rohrer Schnidrig1, Thomas Parkel ${ }^{3}$, Ioannis Stergiou ${ }^{3}$, Ye Zhang ${ }^{1}$, Marta Peroni ${ }^{1}$, Sairos Safai ${ }^{1}$, Damien Charles Weber ${ }^{1,4,5}$, Antony Lomax ${ }^{1,2}$, Giovanni Fattori ${ }^{1}$

1 Paul Scherrer Institute, Center for Proton Therapy, Villigen, Switzerland

2 Department of Physics, ETH Zurich, 8092 Zurich, Switzerland

3 Swiss Center for Electronics and Microtechnology (CSEM), Landquart, Switzerland

4 Department of Radiation Oncology, University Hospital Zurich, 8091 Zurich, Switzerland

5 Department of Radiation Oncology, University Hospital Bern, 3000 Bern, Switzerland

\begin{abstract}
In this study, a functioning and ventilated anthropomorphic phantom was further enhanced for the purpose of CT and MR imaging of the lung and liver. A deformable lung, including respiratory tract was 3D printed. Within the lung's inner structures is a solid region shaped from a patient's lung tumour and six nitro-glycerine capsules as reference landmarks. The full internal mesh was coated, and the tumour filled, with polyorganosiloxane based gel. A moulded liver was created with an external casing of silicon filled with polyorganosiloxane gel and flexible plastic internal structures. The liver, fitted to the inferior portion of the right lung, moves along with the lung's ventilation. In the contralateral side, a cavity is designed to host a dosimeter, whose motion is correlated to the lung pressure. A 4DCT of the phantom was performed along with static and 4D T1 weighted MR images. The CT Hounsfield units (HU) for the flexible 3D printed material were -600 - 100HU (lung and liver structures), for the polyorganosiloxane gel 50 - 120HU (lung coating and liver filling) and for the silicon 650 - 800HU (liver casing). The MR image intensity units were $0-150,0-100$ and $80-110$ respectively. The maximum range of motion in the 4D imaging for the superior lung was 1 $-3.5 \mathrm{~mm}$ and $3.5-8 \mathrm{~mm}$ in the inferior portion. The liver motion was $5.5-8.0 \mathrm{~mm}$ at the tip and 5.7 $-10.0 \mathrm{~mm}$ at the dome. No measurable drift in motion was observed over a two hour session and motion was reproducible over three different sessions for $\sin ^{2}(t), \sin ^{4}(t)$ and a patient-like breathing curve with the interquartile range of amplitudes for all breathing cycles within $0.5 \mathrm{~mm}$. The addition of features within the lung and of a deformable liver will allow the phantom to be used for imaging studies such as validation of 4DMRI and pseudo CT methods.
\end{abstract}




\section{Introduction}

Organ motion adversely affects the accuracy of radiation therapy (Shirato et al., 2004; Seco et al., 2008; Rosu et al., 2003; Zhang et al., 2012) particularly for treatments in the lung, liver and pancreas, where patients' breathing leads to significant intra-fraction anatomical changes. For this reason motion mitigation strategies have to be applied and, recently, the concepts of real-time adaptation during treatment delivery have been explored (Zhang et al., 2016; van de Water et al., 2009) and implemented (Verellen et al., 2010; Poulsen et al., 2015; Caillet et al., 2017). The role of non-ionising imaging techniques to facilitate these treatments is of interest due to the lower dose to the patient. These include direct imaging on MR Linacs (Menten et al., 2016) as well as the use of ultrasound (Harris et al., 2010; Sawada et al., 2004) or optical tracking (Fassi et al., 2014) as a surrogate for target motion along with correlation models (Berbeco et al., 2005). Motion models built using non-ionising imaging techniques often rely on deformable image registration (DIR) techniques which come with their own challenges (Ribeiro et al., 2018; Kashani et al., 2008) with respect to quality assurance and benchmarking. Digital and physical phantoms are valuable tools for the verification and validation of such techniques and their clinical uses.

Physical phantoms are useful to assess complex imaging techniques and their applications in endto-end tests performed under clinical conditions. Many imaging phantoms designed for 4D thorax imaging are simple geometric phantoms with moving targets such as commercial phantoms (e.g. QRM Sim4D+Thorax-Phantom (QRM GmbH, Moehrendorf, Germany), Respiratory Gating platform (Standard Imaging Inc., Middleton, WI, USA) and MODUS-QA quasar respiratory motion phantom (Modus Medical Devices Inc., London, CA)) or custom phantoms (Fattori et al., 2016; Schneider et al., 2019) generally driven by step motors. Others are anthropomorphic (Mayer et al., 2015; Steidl et al., 2012; Serban et al., 2008; Nioutsikou et al., 2006; Followill et al., 2007; Hazelaar et al., 2018), such as CIRS (Dynamic Thorax Phantom-CIRS Inc., Norfolk, VA, USA) but with only a moving target, these are not necessarily useful for validating all 4D imaging techniques. Some phantoms are intended mainly for imaging purposes such as those relying on porcine lungs (Mann et al., 2017) which provide realistic lung images (though the surrounding thorax does not move) but are not convenient or reproducible over the long term. Other phantoms also offer the possibility to include dosimeters for dose measurements (Pallotta et al., 2018; Perrin et al., 2017; Nioutsikou et al., 2006). One commercial phantom, the Dynamic Anatomical Respiring Humanoid Phantom (Radiological Support Devices Inc., Long Beach, CA, USA) and some custom phantoms encompass both imaging and dosimetry (Steidl et al., 2012; Perrin et al., 2017) but do not suit 4DMRI imaging and DIR of the lung as they miss clear features within the lung.

In this study, we describe extensions and improvements on an existing anthropomorphic thorax phantom (Perrin et al., 2017) used at our institute for 4D dosimetry and benchmarking of new motion mitigation techniques (Gorgisyan et al., 2019; Ehrbar et al., 2016). The phantom is equipped with skin, rib cage, heart and a lung model, and mimics the anatomy of a patient thorax. The lung's motion, which follows a breathing pattern predetermined and driven via a ventilation system, moves the rib cage, and a tumour target located inside a cylinder in the left lung portion. Such a simplified design makes manufacturing easier but limits the phantom capabilities for systematic investigation of baseline drift effects or, more in general, complex correlations between the target 
and the external surface motions. Moreover, the realism of the lung filling is limited as it consists of a homogeneous foam material with density close to air, while a patient lung has roughly a third of the density of water, and has many branching structures made up of blood vessels and airways.

Building on our experience with the first iteration, and considering the new requirements for MRguided radiation therapy of lung and liver tumours, such as the need for complex deformable structures, which have contrast in both CT and MR, we report here on further developments of this phantom focusing on:

- Manufacturing of a deformable, anatomically correct, artificial liver

- Digital modelling and fabrication of a new lung, designed to mechanically follow a patient's breathing motion, and mimicking the imaging texture of parenchyma

- Installation of an independent motion controller for the lung tumour target

The described solution includes a new lung model with increased tissue contrast, allowing for the validation of 4DMR imaging techniques and their use for the generation of simulated 4DCT data sets based on extracted motions (Boye et al., 2013; Bernatowicz et al., 2016). Both the lung and liver components have visible features in the CT, and distinguishable structures in T1-weighted MRI images. In this study, methods used in upgrading the phantom are outlined and the new lung and liver assessed for both motion and image contrast characteristics in advanced MR imaging.

\section{Methods}

\section{Phantom development}

\section{Liver component}

The artificial liver is a deformable replica of a three-dimensional anatomical model of the organ commercialised by Erler Zimmer GmbH \& Co. KG (Lauf, Germany) as an educational tool. Its manufacturing (Fig. 1) starts with the creation of a negative mould of the object by immersing the plastic model in an silicon (Elastosil M4601) bath that thicken over 24-48 hours. A placeholder was made from modelling clay, Fimo, and placed inside the mould to provide a negative space inside the external liver component casing, to be filled with a more flexible substance. The casing of the liver was made by filling the mould with liquid silicon (RTV Silikon 1703, Suter Kunststoffe). Prior to pouring the silicon mixture, a vacuum pump was used to remove air bubbles in order to avoid imaging artefacts caused by air. Finally, the liver was filled with polyorganosiloxane-based gel (Raytech SRL, Settimo Milanese, Italy) to provide greater flexibility of the component as well as imaging contrast to the external casing. Small inserts of Visijet M2 EBK deformable 3D printing material (3DSystems, South Carolina, USA) printed using a Project MJP 2500 Plus printer (3DSystems, South Carolina, USA) were suspended in the gel filling, to provide vein-like structures within the liver, two of approximate $3 \mathrm{~cm}$ length and two of $1.5 \mathrm{~cm}$ ranging non-uniformly in diameter between $2-5 \mathrm{~mm}$. 


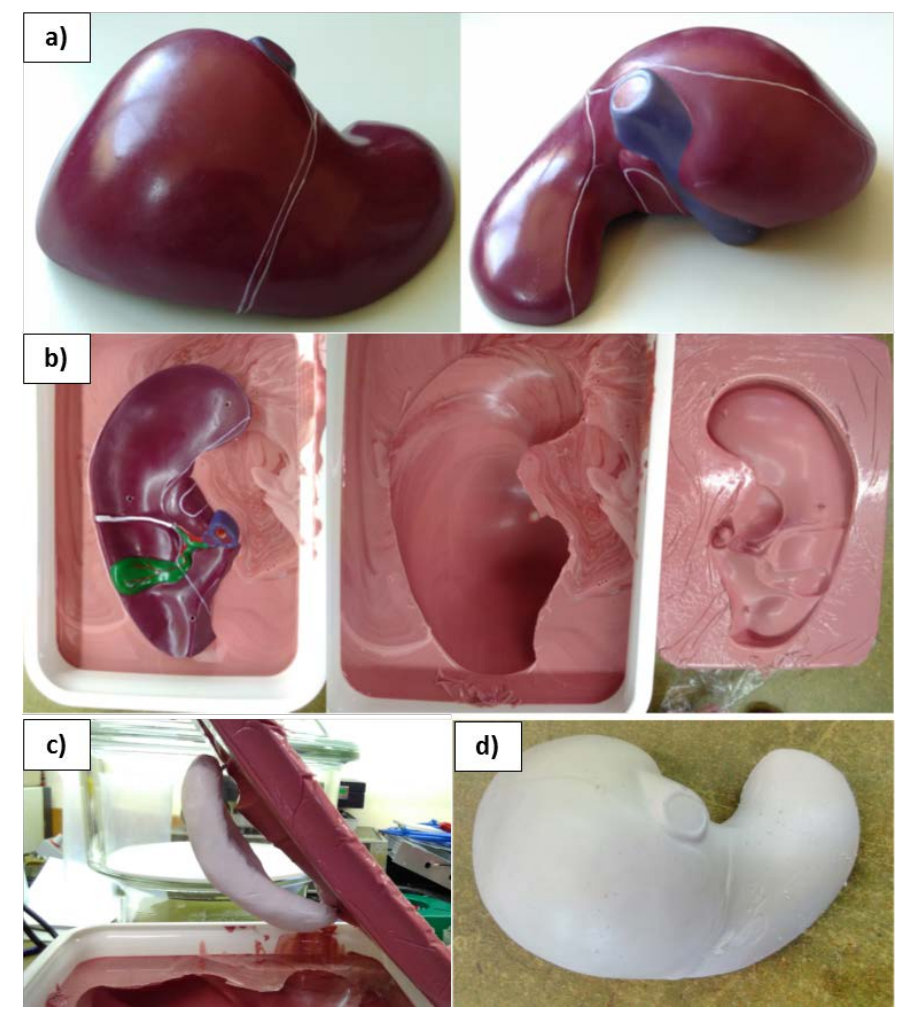

Figure 1, The manufacturing workflow of the liver component: a) anatomical liver model b) creation of the negative cast c) Liver mould with internal placeholder d) final liver component with silicon casing and polyorganosiloxane-based gel filling and internal plastic features

\section{$3 D$ printed lung}

The design and printing of the lung was performed by creating a three-dimensional digital model featuring a lung tumour segmented from a patient CT using Velocity (version 3, Varian Medical Systems, Palo Alto, CA) and the respiratory tract of a real patient (Thorax CT patient 001, supplemental data (Reynisson et al., 2015)), segmented up to the third bifurcation. 3D computer models of the thorax bone structure, the liver phantom, a lung tumour and respiratory tract were utilised in the design of the phantom lung, filling the anthropomorphic thorax phantom's cavity (Fig. 2). Its internal volume was filled with structures designed to provide the mechanical properties needed for the respiratory motion of the lung, and additionally mimic the lung's parenchyma tissue in section view (Fig. 3). The structure is a cubic structure of cylinders (lattice), and the cell's dimensions are $15 \times 15 \times 15 \mathrm{~mm}^{3}\left(225 \mathrm{~mm}^{3}\right)$.

The patient tumour, placed in the centre of the right side of the lung has increased density compared to the surrounding area, and was fully filled with polyorganosiloxane based gel during the printing process to enhance MR visibility. On the other side, the left side of the lung was modelled with a cut-out cylindrical cavity allocated for dosimetry purposes (see paragraph Independent lung tumour motion controller). Finally, the inferior portion of the lung (diaphragm) on the right-hand side was shaped to fit and hold in place the artificial liver. 


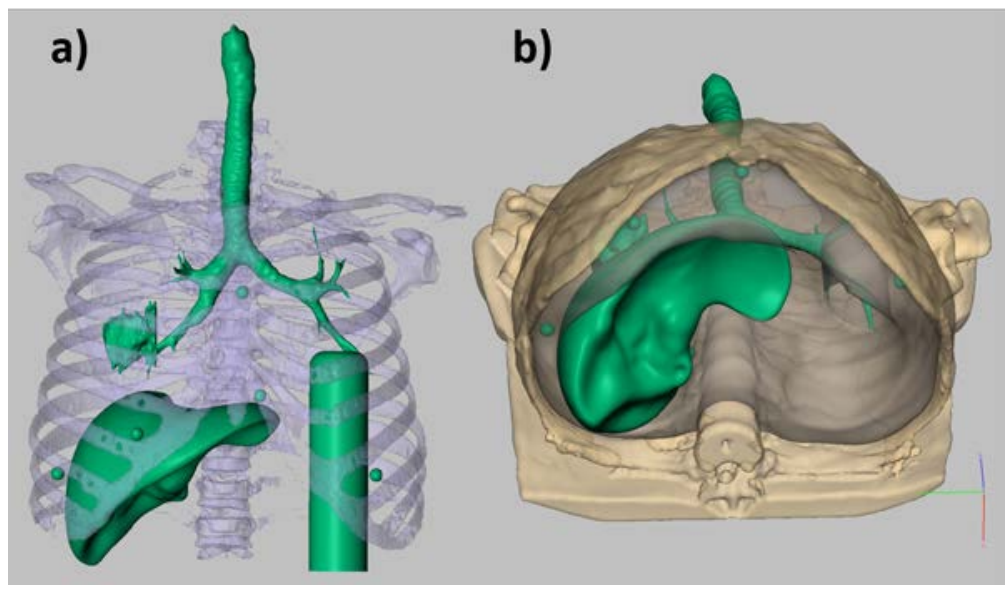

Figure 2, a) All 3D models, b) that resulted in shaping the lung for the existing anthropomorphic thorax phantom

The lung has been 3D printed using a fused-deposition-modeling (FDM)-based 3D printer FORMBOT T-Rex 2+ (Jinhua Xinke 3DTechnology CO. LTD, China) with a flexible Thermoplastic polyurethane (TPU) filament, that is graded with a shore hardness of 85 on the A scale. The accuracy of the printing of this material is within $0.5 \mathrm{~mm}$, as per specifications. The full interior of the model was coated with polyorganosiloxanes-based gel agitating the phantom to evenly disperse a small amount of material poured through the trachea. Such a thin gel layer is a stable compound that provides enough oxygen for MR imaging contrast. Elastic polyurethane (PUR) coating was applied on the outer surface to ensure that the structure is airtight. During printing, six nitroglycerin capsules of approx. $8.8 \mathrm{~mm}$ in diameter were placed within the lung for use as landmarks, as they can be easily seen on MR and CT images.
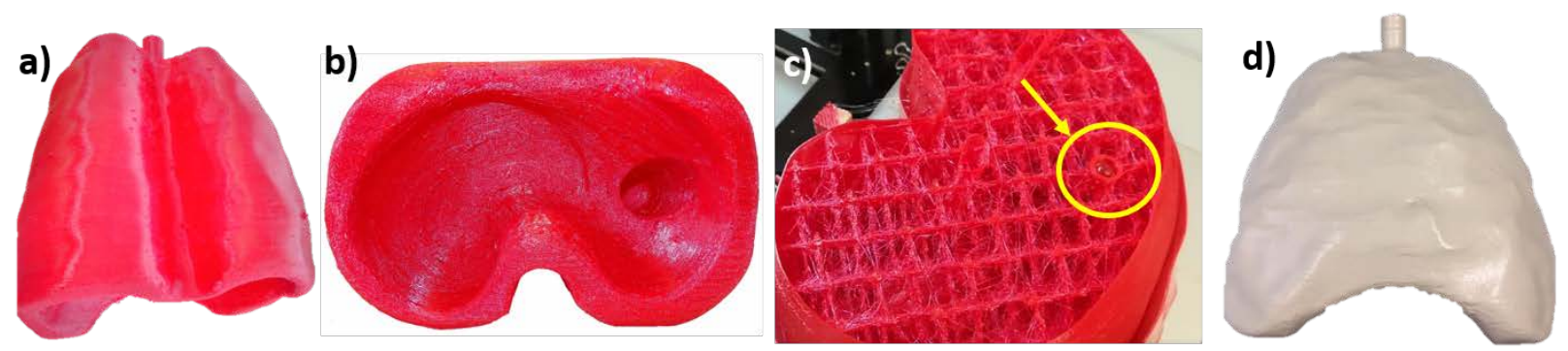

Figure 3, a) the 3D printed lung from the posterior view, b) inferior portion (diaphragm) shaped to fit the artificial liver on the right hand side, and c) the interior during printing with a nitro-glycerine capsule placed on the right hand side, indicated with the yellow arrow d) the final coated lung

\section{Independent lung tumour motion controller}

The motion controller is independent to the main part of the thorax phantom (Fig 4.) and moves a dosimetry target in the space in the inferior portion of the lung component. It is designed to execute realistic internal-external correlation patterns observed in patients, typically characterized by shifts in phase and baseline over time. As such, a holder, for dosimetry purposes, has been mounted on an aluminium rod sliding through a linear stage fixed on the phantom support plate. Based on the Arduino EDISON breakout board, the processing units (Honeywell SSCDRRN060MDAA5) reads 
the lung pressure from a derivation of the inlet pipe to set in real time the absolute angular position of a servo motor (Dynamixel MX-28R) using the equation below:

$$
\alpha(P)=P \cdot \text { gain }+ \text { offset }
$$

Where $\alpha(\mathrm{P})$ is the angular position, $\mathrm{P}$ is the pressure, the gain refers to the ratio of the pressure magnitude to the angular position. Speed modulation was applied to preserve motion smoothness over a wide range of input pressures. Specifically, the first derivative of the pressure trace is computed over a short history of the signal and a least square algorithm used to proportionally correct the motor's shaft rotating speed.

A pulley translates the rotational motion of the servo motor into a linear displacement of the dosimetry holder and its size defines the achievable range of motion. The controller has been tested operating the phantom under different motion settings to assess the actuation latency. For convenience, a small cantilever has been solidly fixed on the motor shaft to ease the observation of motion output and compare it with the lung expansion using a third party optical position sensor (Polaris SPECTRA, NDI). Two passive infrared markers were used for this measurement, fixed respectively on the tip of the motor shaft and the superior surface of the lung phantom. The latency of the motion controller was quantified by assessing the delay between the lung surface displacement, considered as surrogate of the lung ventilation, and the corresponding position update of the target. By ventilating the model with a sinusoidal breathing waveform, and subsequent Fourier analysis, phase shifts between the two signals (input and measured) could be estimated.

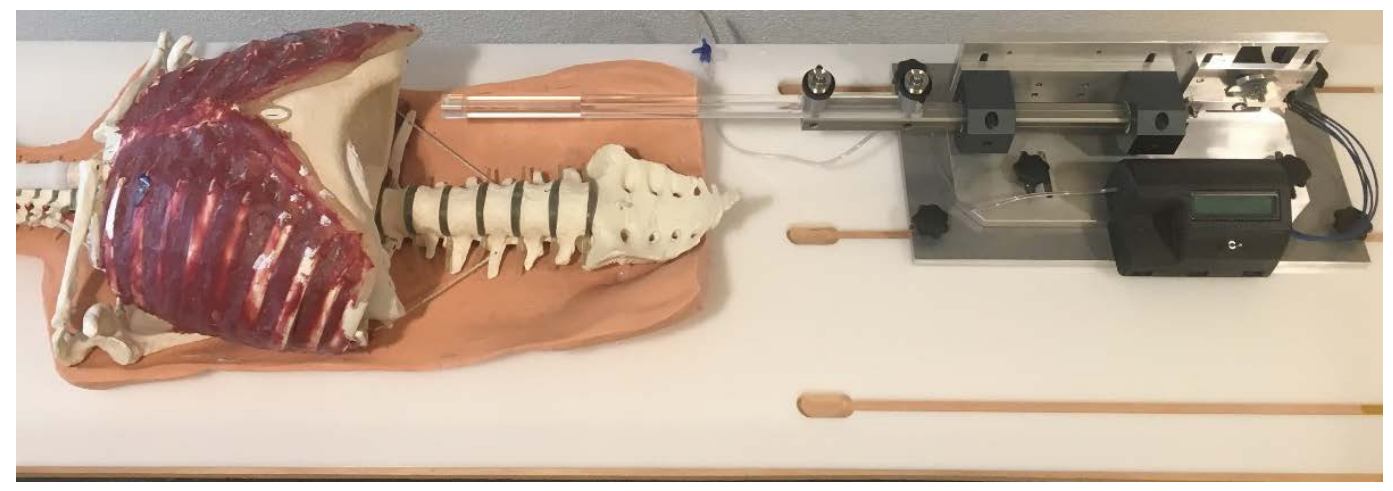

Figure 4, The anthropomorphic phantom (without the upper silicon skin) with new lung and liver components and on the right hand side the independent lung tumour controller

\section{Lung and liver motion assessment}

In order to assess the motion qualities of the lung and liver components, CT and MR images were obtained with and without ventilation. The breathing motion applied by the ventilating system to the lung followed $\sin ^{4}(\mathrm{t})$ pattern with a period of 4 seconds with a pressure range from $-5 \mathrm{mBar}$ to 25 mBar.

The 4DCT images were acquired, using the clinical protocol (mAs: 80, kVp:120, CARE Dose4D), on a Siemens Somatom Sensation Open CT on sliding rails with $0.9766 \mathrm{~mm}$ in-plane resolution and 2 
mm slice thickness, in cranio-caudal direction with a CTDI $_{\mathrm{vol}}$ of 16-19 mGy. In total, 8 respiratory phases, sorted in amplitude were reconstructed retrospectively using the respiratory surrogate signal measured with the low-sensitivity load cell of the Anzai Gating System AZ-733 V (Anzai Medical Co., Ltd, Tokyo, Japan).

Under the same motion settings, MR images were acquired using a Siemens Aera 1.5T MRI. For the 4DMRI, an interleaved navigator-based retrospectively sorted sequence described by (von Siebenthal et al., 2007) was used with parameters: TE: $0.95 \mathrm{~ms}$, TR: $149.51 \mathrm{~ms}$, flip angle: 49, resolution: $2.08 \mathrm{~mm}$, slice thickness: $5 \mathrm{~mm}$, slice spacing: $3.35 \mathrm{~mm}$, acquisition direction: sagittal. After reconstruction this 4DMR sequence results in a series of 3D volumes, at frequency of $3.33 \mathrm{~Hz}$, created from stacked 2D sagittal images. Static images were acquired at end exhale and full inhale using an abdominal T1 weighted sequence with parameters: TE: $11 \mathrm{~ms}$, TR: $768 \mathrm{~ms}$, flip angle: 150, resolution: $0.4883 \mathrm{~mm}$, slice thickness: $3.5 \mathrm{~mm}$, slice spacing: $4.375 \mathrm{~mm}$, acquisition direction: coronal.

In order to quantify the overall range of motion within the phantom, a B-Spline based deformable image registration (Velocity ${ }^{\mathrm{TM}}$, Varian Medical Systems, Inc) was performed between the end-exhale and full-inhale phases of the 4DCT, the static MRIs and 4DMRI. The maximum and minimum magnitudes of the deformable vector fields were calculated for different regions of the lung and liver between the exhale and inhale of all three data sets.

The drift in phantom motion after cold start has been assessed over two hours by tracking the position of the inferior nitro-glycerine marker in the lung over the phases of 4DCT images (using the same protocol described above) with deformable image registration. The measured range of motion at the session start is compared with the marker displacement after one and two hours of continuous breathing with sinusoidal pattern. In addition, the day-to-day reproducibility has been assessed on sagittal 2D cine MR images with $1.28 \mathrm{~mm}$ pixel spacing acquired at $5 \mathrm{~Hz}$. The in-plane displacement of a central marker has been measured ventilating the phantom in the $(-5,20) \mathrm{mBar}$ pressure range for three different motion curves, $\sin ^{2}(t)$ and $\sin ^{4}(t)$ with 4 second period and a patient breathing trajectory with average period 6 seconds and variability in amplitude of $15 \%$. Results are reported for three measurements over two weeks and the median and interquartile range (IQR) of the all breathing cycles for each motion type was calculated.

\section{Image contrast assessment}

The image contrast of the different materials used in the construction of lung and liver components was assessed on the 4DCT, the static MRI and 4DMRI images described above. The given HU and MR intensity units for the silicon are the range of values within eight regions of interest (ROIs) of 20x20 pixels (CT: $19.5 \times 19.5 \mathrm{~mm}$, MR: $9.8 \times 9.8 \mathrm{~mm}$ ) on 4 slices ( $8 \mathrm{~mm}$ for CT and $14 \mathrm{~mm}$ for MR). For the polyorganosiloxane gel, five, $10 \times 10$ pixel (CT: $9.8 \times 9.8 \mathrm{~mm}$, MR: $4.9 \times 49$. mm) ROIs were defined, on 4 slices within the internal filling of the liver. The 3D printed plastic structures within the liver were assessed using one ROI of 5x5 pixels (CT: $4.9 \times 4.9 \mathrm{~mm}$, MR: $2.4 \times 2.4 \mathrm{~mm}$ ) on 4 slices and 30 ROIs of $1 \times 1$ pixels (CT: $0.98 \times 0.98 \mathrm{~mm}$, MR: $0.49 \times 0.49 \mathrm{~mm}$ ). The 3D printed material of the lung, which was printed thickness of approximately $1.6 \mathrm{~mm}$, was assessed in the area at the top of the respiratory tract, in the $\mathrm{CT}$, which was not coated in polyorganosiloxane gel, and surrounding the 
tumour in the MRI using 30 ROIs of $1 \times 1$ pixels. Background air values were taken using six ROIs of 30x30 pixels on 4 slices (CT: $39.1 \times 39.1 \mathrm{~mm}$, MR: $14.6 \times 14.6 \mathrm{~mm}$ ) in the space below the liver compartment and above the shoulders. The Hounsfield units were extracted from the CT images, and MR intensities of the lung and liver, relative to air, were assessed in the static MR and 4D MR images. Additionally, the HU and MR intensity units of patient images using the same imaging protocols were also assessed for the lung and liver.

\section{Results}

\section{Phantom development}

The 3D printed lung fits in to the original phantom rib cage attached to a base plate and is designed so that the moulded liver compartment fits into the bottom of the lung (Fig. 4). The independent tumour controller (used to control a dosimetry tumour, rather than the internal imaging tumour) is attached inferior to the phantom aligned with the dosimetry cavity on the left phantom lung. The dosimeter motion controller, which is not MR compatible, can be easily removed for MR imaging.

The pulley of the independent tumour controller has a $30 \mathrm{~mm}$ diameter, allowing for a maximal 30 mm motion range for the dosimeter. Through comparison of the lung surface motion curve and that of the target position, the latency was measured to be on average $77.67 \pm 17.52 \mathrm{~ms}$.

\section{Lung and liver motion}

The motion between the full inhale and full exhale static MR volumes in the superior and posterior portions of the lung was primarily in the anterior direction with a range of $1-3.5 \mathrm{~mm}$ (Fig 5). The inferior lung was seen to move in an anterior-inferior direction of approximately $45^{\circ}$ with a range of 3.5 - $8 \mathrm{~mm}$. The right-lung tumour lesion moved 3.5-6.0 mm mostly in the AP direction with the bottom of the target moving more than the top showing that there is some deformation of the target itself. Motion of the liver is generally in the inferior direction with a smaller amount of anterior motion. Motion of the ranges of $5.5-8.0 \mathrm{~mm}$ were observed at the tip and $5.7-10.0 \mathrm{~mm}$ at the lobe of the liver phantom; posteriorly the motion is slightly smaller with $2.5-3 \mathrm{~mm}$. 

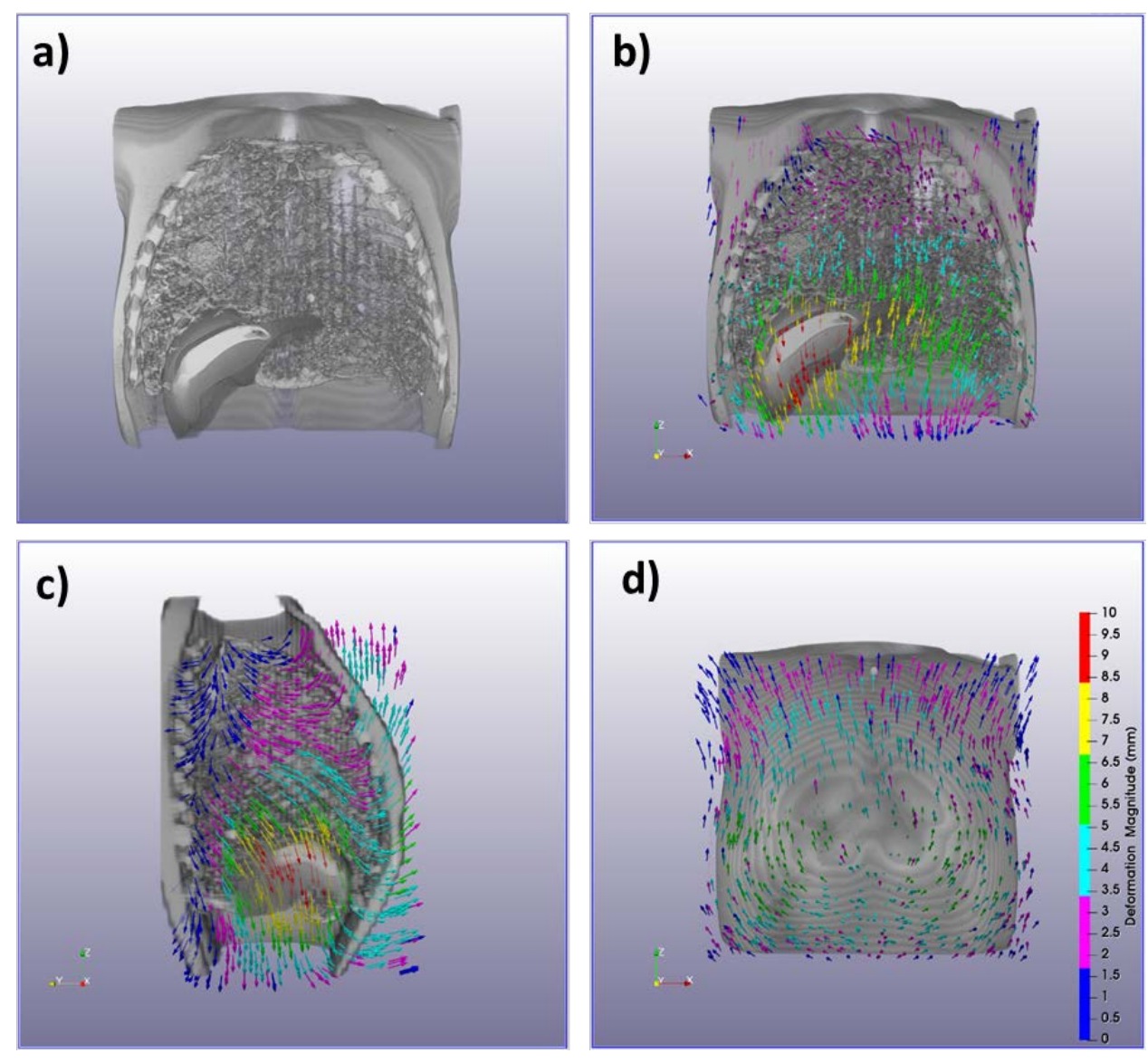

Figure 5: a) 3D rendering of the MR image volume of the phantom in a coronal slice (b), sagittal slice (c) and anterior surface (d). The motion vector field from the inhale to exhale phases of the static MR is overlaid.

The 4DMRI reconstruction was performed and the resultant stacked 3D images assessed; no obvious artefacts were seen in the stacking of the 2D images. Figure $6 \mathrm{c}$ ) shows a coronal view of a 3D stack representing the inhale phase of a single breathing cycle of the 4DMRI. The motion between end-exhale and full-inhale breathing phases of the reconstructed 4DMRI and 4DCT agrees to MRs acquired holding the phantom stationary in the respective states (Fig. 5) with maximum and minimum magnitude of vector fields within $1 \mathrm{~mm}$ for each of the regions (superior lung, posterior lung, inferior and anterior lung, tip and lobe of liver).

The repeated 4DCTs over a two hour session ventilating the phantom with a sinusoidal driving curve $\left(\sin ^{4}\right)$ showed remarkable motion reproducibility with the most inferior marker consistently moving by $2.5 \mathrm{~mm}$ range. Similarly, DIR between corresponding breathing phases in subsequent acquisitions did not show systematic drifts in end-exhale and full-inhale lung states with $<0.1 \mathrm{~mm}$ difference in the three-dimensional vector fields. Day-to-day variations were assessed on sagittal MR images aligned at the inferior central nitro-glycerine marker for sinusoidal $\left(\sin ^{2}(t), \sin ^{4}(t)\right)$ and patient-like breathing curve. The median and IQR of the marker motion amplitude for the cycles imaged over three imaging sessions were $1.7 \pm 0.2 \mathrm{~mm}$ (24 cycles), $1.5 \pm 0.4 \mathrm{~mm}$ (25 cycles) and 1.3 $\pm 0.4 \mathrm{~mm}$ (13 cycles) respectively. 


\section{Material contrast}

The materials used for construction of the lung and liver components each provide contrast in both CT and MR images (Fig 6). The T1 MR intensity values, taken from the static MR, relative to air and the CT HU for the 3D printed plastic structures inside the liver and forming the lung structure, the polyorganosiloxane gel and the silicon encasing the liver along with those of a corresponding patient image are shown in Table 1. The lung 3D printed material was thin and coated in polyorganosiloxane gel in most places, for this reason 30 single pixel ROIs were used and the calculated mean (standard deviation) were -115 (53) HU and 11 (10.6) MR intensity units. The T1 MR intensity units relative to air obtained from the 4DMRI images were in the same range for the two 3D printed materials but lower for the silicon casing and the Polyorganosiloxane gel with 10-30 and 50-110 respectively.

Table 1. The mean (and range) of the raw CT Hounsfield Units and T1 MR intensity values relative to air (from static MR) for the materials used in construction along with those for patient anatomy

\begin{tabular}{|lll|}
\hline Material & HU & T1 MR intensity \\
\hline Phantom materials & & \\
\hline Lung 3D printed TPU & $-115(-200--50)$ & $11(0-40)$ \\
\hline Liver Visijet M2 EBK & $-203(-600-100)$ & $13(0-25)$ \\
\hline Silicon casing & $735(650-800)$ & $108(80-130)$ \\
\hline Polyorganosiloxane gel & $68(30-120)$ & $249(210-280)$ \\
\hline Patient anatomy & & \\
\hline Lung & $-880--110$ & - \\
\hline liver & $35-230$ & - \\
\hline
\end{tabular}



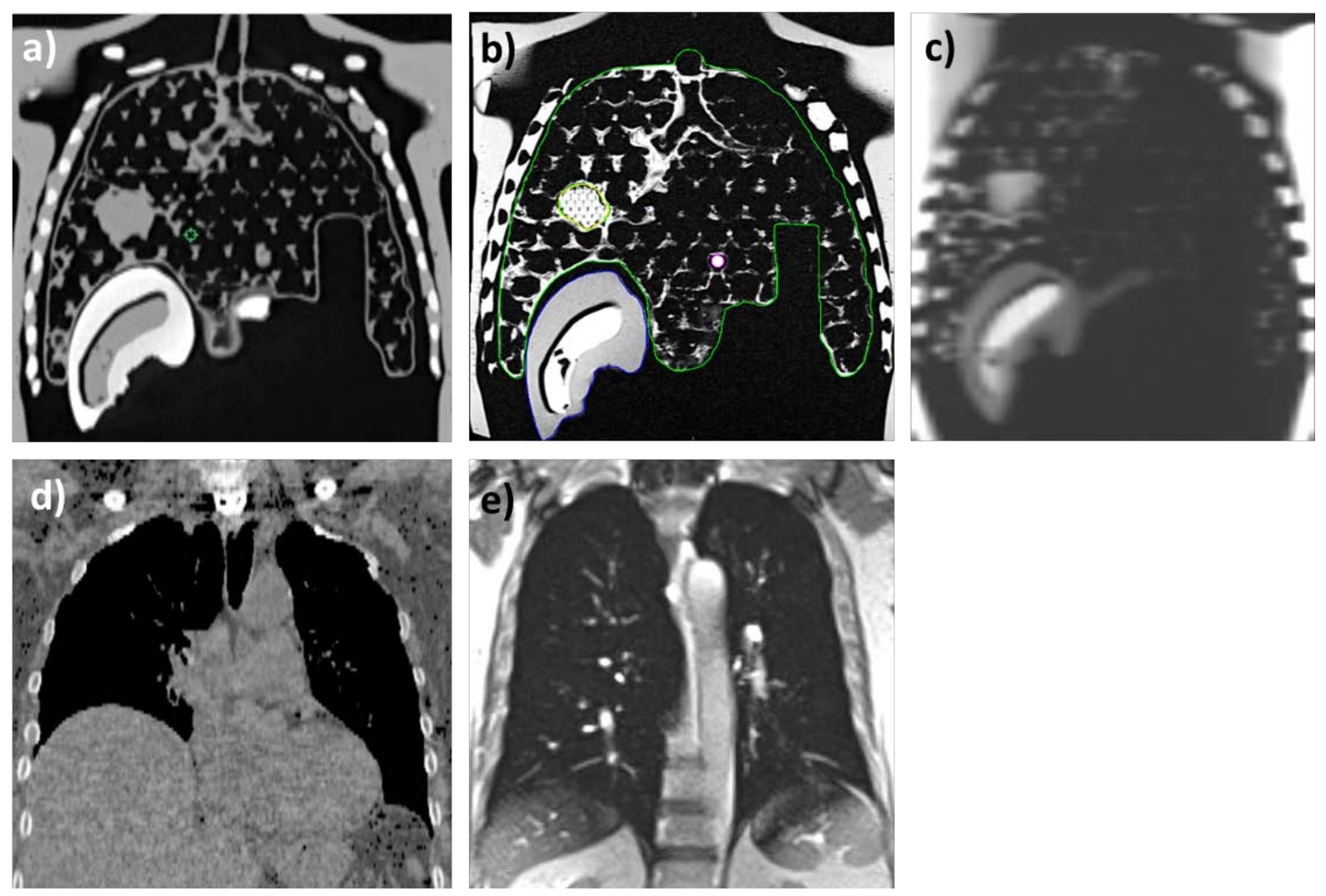

Figure 6, a) coronal view of a single phase of the 4DCT image, b) coronal view of T1 MRI scan with the lung (green), liver(blue), imaging target(yellow) and the nitro-glycerine marker(purple) contoured, and c) the coronal view of a single 4DMRI volume (stack of 2D sagittal slices) representing inhale d) a patient 4DCT single phase image e) single slice slice of a coronal patient 4DMRI

\section{Discussion}

In this study a dynamic anthropomorphic thorax phantom has been modified to allow enhanced MR and CT imaging of lung and liver. The image qualities of the lung and liver additions have been investigated along with the nature of the motion present. While the motion of the inner tumour target of this phantom is not as large as many other geometric or anthropomorphic phantoms, the complexity of the motion and deformation of other portions of the phantom (such as ribs liver and lung structures) is greater, increasing the usefulness of this phantom for imaging studies involving deformable image registration. Moreover, on the left-side inferior surface of the lung is a space for a dosimetry target which moves independent of, but correlated with, the ventilation of the lung itself.

Selection of a material for use in a 3D printing of the lung that is flexible is a challenge, as 3D printing materials are usually rigid and not flexible enough for our intended purposes. To enhance the lung's deformability, a designated structural pattern design, inside the lung, was applied. The flexible TPU filament was selected because of its flexibility even though it is not visible in MR images. Due to this trait, different structures are prominent on the CT and MR images, such as internal structures of the tumour (Fig 6). 
During the manufacture of the liver component, several challenges were encountered including difficulty with air bubbles in the silicon used for the external casing, which were successfully overcome using a vacuum pump. The thickness of the casing was also not consistent, which resulted in leakage of air into the component. This inconsistent case thickness was due to the challenge of creating the internal placeholder using moulding clay. The materials used for the liver are compatible with US imaging (Maggi et al., 2009) and it is possible to find features in the liver when the probe was placed directly on the liver itself, which suggests feature motion tracking will be possible in the future.

The range of motion of the lung and liver components in the phantom (10 $\mathrm{mm}$ ) are smaller than the average amplitude of the diaphragm for patients $(16.4 \mathrm{~mm}$ ) (Rit 2012). However, the motion is not unrealistically small for a patient and the amplitude and deformation of the components are adequate for use with 4DMRI and DIR.

This is the first version of the updated phantom with 3D printed flexible components, and therefore durability and resistance is still to be seen over the long term. However, the polyorganosiloxane gel has an intended use in insulation of electrical circuits for which it is claimed to have an indefinite shelf life. The 3D printed structures within the liver were made using Visijet M2 EBK (3DSystems, South Carolina, USA) which is a rubbery elastic type substance, and these were encased in flexible polyorganosiloxane gel. Mechanical durability of the lung model has been observed during the manufacturing process. The 3D printed lung stucture itself is covered externally by a sealant and internally coated in polyorganosiloxane gel, meaning there will be very little exposure to light or air.

As the intention of the addition of the lung and liver were to develop the existing phantom primarily into an imaging phantom, focusing on motion and deformation, it was not a requirement for our institute to use tissue equivalent materials. The materials were selected rather to ensure image contrast in MR and CT imaging rather than replicate the density of human tissues. This is evident with the 3D printed lung material and the polyorganosiloxane gel having higher HU than that of lung tissue. If the research activity utilising the phantom was to begin to focus on noise and lesion detection or dosimetry, then the tissue equivalence would be of larger concern and could be improved in future iterations using more sophisticated 3D printed materials (Bustillo et al., 2019). Various 3D printed anthropomorphic phantoms exist for the purpose of medical imaging (Hazelaar et al., 2018; Filippou and Tsoumpas, 2018), a quickly expanding field of research (Tino et al., 2019). Some phantoms have image contrast very similar to that seen in CT (Hernandez-Giron et al., 2019; Hazelaar et al., 2018; Zhang et al., 2019; Bustillo et al., 2019) and MR (Wake et al., 2019; He et al., 2019).

The motion controller for the independent dosimetry lung tumour, which is correlated with the pressure of the ventilated lung with a slight latency, will allow for the end-to-end use of the phantom through imaging and treatment delivery. The motion range of the target is currently 30 $\mathrm{mm}$ but there is the possibility of increasing this with the change of the pulley to one with a larger diameter. 
The developments to the existing anthropomorphic phantom outlined in this manuscript, including the addition of a 3D printed lung, moulded liver component and independent tumour, means that it is now compatible with CT, MR, Ultrasound and optical surface imaging. This allows for validation of advanced image processing procedures such as DIR, combining different imaging techniques, and their applications, along with dosimetry assessment in the radiation therapy workflow.

\section{Acknowledgments}

Dr Colvill has received funding from the European Union's Horizon 2020 research and innovation programme under the Marie Skłodowska-Curie grant agreement No 701647. This research was also partially funded under Swiss National Science Foundation grant no. 320030_163330/1. The authors would like to thank CSEM for partially funding this project. Many thanks to the Biomedical Engineering Group of Philippe Cattin at University of Basel for the use of their ultrasound device.

\section{References}

Berbeco R I, Nishioka S, Shirato H, Chen G T Y and Jiang S B 2005 Residual motion of lung tumours in gated radiotherapy with external respiratory surrogates Physics in Medicine and Biology $\mathbf{5 0}$ 3655-67

Bernatowicz K, Peroni M, Perrin R, Weber D C and Lomax A 2016 Four-dimensional dose reconstruction for scanned proton therapy using liver 4DCT-MRI International Journal of Radiation Oncology* Biology* Physics 95 216-23

Boye D, Lomax T and Knopf A 2013 Mapping motion from 4D-MRI to 3D-CT for use in 4D dose calculations: A technical feasibility study Medical physics $\mathbf{4 0}$

Bustillo J P, Tumlos R and Remoto R Z World Congress on Medical Physics and Biomedical Engineering 2018, (Singapore, 2019// 2019), vol. Series) ed L Lhotska, et al.: Springer Singapore) pp 509-15

Caillet V, Keall P J, Colvill E, Hardcastle N, O'Brien R, Szymura K and Booth J T 2017 MLC tracking for lung SABR reduces planning target volumes and dose to organs at risk Radiotherapy and Oncology 124 18-24

Ehrbar S, Perrin R, Peroni M, Bernatowicz K, Parkel T, Pytko I, Klöck S, Guckenberger M, Tanadini-Lang S, Weber D C and Lomax A 2016 Respiratory motion-management in stereotactic body radiation therapy for lung cancer - A dosimetric comparison in an anthropomorphic lung phantom (LuCa) Radiotherapy and Oncology 121 328-34

Fassi A, Schaerer J, Fernandes M, Riboldi M, Sarrut D and Baroni G 2014 Tumor Tracking Method Based on a Deformable 4D CT Breathing Motion Model Driven by an External Surface Surrogate International Journal of Radiation Oncology*Biology*Physics 88 182-8

Fattori G, Seregni M, Pella A, Riboldi M, Capasso L, Donetti M, Ciocca M, Giordanengo S, Pullia M, Marchetto F and Baroni G 2016 Real-time optical tracking for motion compensated irradiation with scanned particle beams at CNAO Nuclear Instruments and Methods in Physics Research Section A: Accelerators, Spectrometers, Detectors and Associated Equipment 827 39-45

Filippou V and Tsoumpas C 2018 Recent advances on the development of phantoms using 3D printing for imaging with CT, MRI, PET, SPECT, and ultrasound Medical Physics 45 e740-e60

Followill D S, Evans D R, Cherry C, Molineu A, Fisher G, Hanson W F and Ibbott G S 2007 Design, development, and implementation of the Radiological Physics Center's pelvis and thorax anthropomorphic quality assurance phantoms Medical Physics 34 2070-6

Gorgisyan J, Lomax A J, Rosenschold P M a, Persson G F, Krieger M, Colvill E, Scherman J, Gagnon-Moisan F, Egloff M, Fattori G, Engelholm S A, Weber D C and Perrin R 2019 The dosimetric effect of 
residual breath-hold motion in pencil beam scanned proton therapy - An experimental study Radiotherapy and Oncology 134 135-42

Harris E J, Miller N R, Jeffrey C B, Symonds-Tayler J R N and Philip M E 2010 Speckle tracking in a phantom and feature-based tracking in liver in the presence of respiratory motion using $4 \mathrm{D}$ ultrasound Physics in Medicine and Biology 553363

Hazelaar C, van Eijnatten M, Dahele M, Wolff J, Forouzanfar T, Slotman B and Verbakel W F A R 2018 Using 3D printing techniques to create an anthropomorphic thorax phantom for medical imaging purposes Medical Physics 45 92-100

He Y, Liu Y, Dyer B A, Boone J M, Liu S, Chen T, Zheng F, Zhu Y, Sun Y, Rong Y and Qiu J 2019 3D-printed breast phantom for multi-purpose and multi-modality imaging Quant Imaging Med Surg 9 63-74

Hernandez-Giron I, den Harder J M, Streekstra G J, Geleijns J and Veldkamp W J H 2019 Development of a 3D printed anthropomorphic lung phantom for image quality assessment in CT Physica Medica: European Journal of Medical Physics 57 47-57

Kashani R, Hub M, Balter J M, Kessler M L, Dong L, Zhang L, Xing L, Xie Y, Hawkes D, Schnabel J A, McClelland J, Joshi S, Chen Q and Lu W 2008 Objective assessment of deformable image registration in radiotherapy: A multi-institution study Medical Physics 35 5944-53

Maggi L E, Krüger M A v, Pereira W C A and Monteiro E E C 2009 IEEE International Ultrasonics Symposium,20-23 Sept. 2009 2009), vol. Series) pp 1962-5

Mann P, Witte M, Moser T, Lang C, Runz A, Johnen W, Berger M, Biederer J and Karger C P 2017 3D dosimetric validation of motion compensation concepts in radiotherapy using an anthropomorphic dynamic lung phantom Physics in Medicine \& Biology 62573

Mayer R, Liacouras P, Thomas A, Kang M, Lin L and Simone C B 2015 3D printer generated thorax phantom with mobile tumor for radiation dosimetry Review of Scientific Instruments $\mathbf{8 6} 074301$

Menten M J, Fast M F, Nill S, Kamerling C P, McDonald F and Oelfke U 2016 Lung stereotactic body radiotherapy with an MR-linac - Quantifying the impact of the magnetic field and real-time tumor tracking Radiotherapy and Oncology 119 461-6

Nioutsikou E, Symonds-Tayler J R N, Bedford J L and Webb S 2006 Quantifying the effect of respiratory motion on lung tumour dosimetry with the aid of a breathing phantom with deforming lungs Physics in Medicine and Biology 51 3359-74

Pallotta S, Calusi S, Foggi L, Lisci R, Masi L, Marrazzo L, Talamonti C, Livi L and Simontacchi G 2018 ADAM: A breathing phantom for lung SBRT quality assurance Physica Medica 49 147-55

Perrin R L, Zakova M, Peroni M, Bernatowicz K, Bikis C, Knopf A K, Safai S, Fernandez-Carmona P, Tscharner N, Weber D C, Parkel T C and Lomax A J 2017 An anthropomorphic breathing phantom of the thorax for testing new motion mitigation techniques for pencil beam scanning proton therapy Physics in Medicine \& Biology 622486

Poulsen P R, Worm E S, Hansen R, Larsen L P, Grau C and Høyer M 2015 Respiratory gating based on internal electromagnetic motion monitoring during stereotactic liver radiation therapy: First results Acta Oncologica 54 1445-52

Reynisson P J, Scali M, Smistad E, Hofstad E F, Leira H O, Lindseth F, Nagelhus Hernes T A, Amundsen T, Sorger H and Lang $\varnothing$ T 2015 Airway Segmentation and Centerline Extraction from Thoracic CT Comparison of a New Method to State of the Art Commercialized Methods PLOS ONE 10 e0144282

Ribeiro C O, Knopf A, Langendijk J A, Weber D C, Lomax A J and Zhang Y 2018 Assessment of dosimetric errors induced by deformable image registration methods in 4D pencil beam scanned proton treatment planning for liver tumours Radiotherapy and Oncology 128 174-81

Rosu M, Dawson L A, Balter J M, McShan D L, Lawrence T S and Ten Haken R K 2003 Alterations in normal liver doses due to organ motion International Journal of Radiation Oncology*Biology*Physics 57 1472-9 
Sawada A, Yoda K, Kokubo M, Kunieda T, Nagata Y and Hiraoka M 2004 A technique for noninvasive respiratory gated radiation treatment system based on a real time 3D ultrasound image correlation: A phantom study Medical Physics 31 245-50

Schneider S, Dolde K, Engler J, Hoffmann A and Pfaffenberger A 2019 Commissioning of a 4D MRI phantom for use in MR-guided radiotherapy Medical Physics 46 25-33

Seco J, Sharp G C, Wu Z, Gierga D, Buettner F and Paganetti H 2008 Dosimetric impact of motion in freebreathing and gated lung radiotherapy: A 4D Monte Carlo study of intrafraction and interfraction effects Medical Physics 35 356-66

Serban M, Heath E, Stroian G, Collins D L and Seuntjens J 2008 A deformable phantom for 4D radiotherapy verification: Design and image registration evaluation Medical Physics 35 1094-102

Shirato H, Seppenwoolde Y, Kitamura K, Onimura R and Shimizu S 2004 Intrafractional tumor motion: lung and liver Seminars in Radiation Oncology 14 10-8

Steidl P, Richter D, Schuy C, Schubert E, Haberer T, Durante M and Bert C 2012 A breathing thorax phantom with independently programmable 6D tumour motion for dosimetric measurements in radiation therapy Physics in Medicine and Biology 57 2235-50

Tino R, Yeo A, Leary M, Brandt M and Kron T 2019 A Systematic Review on 3D-Printed Imaging and Dosimetry Phantoms in Radiation Therapy Technology in Cancer Research \& Treatment 18 1533033819870208

van de Water S, Kreuger R, Zenklusen S, Hug E and Lomax A J 2009 Tumour tracking with scanned proton beams: assessing the accuracy and practicalities Physics in Medicine and Biology 54 6549-63

Verellen D, Depuydt T, Gevaert T, Linthout N, Tournel K, Duchateau M, Reynders T, Storme G and De Ridder M 2010 Gating and tracking, 4D in thoracic tumours Cancer/Radiothérapie 14 446-54

von Siebenthal M, Szekely G, Gamper U, Boesiger P, Lomax A and Cattin P 2007 4D MR imaging of respiratory organ motion and its variability Physics in medicine and biology $\mathbf{5 2} 1547$

Wake N, lanniello C, Brown R, Walczyk J, Moy L, Collins C and Rusinek H 2019 A 3D printed patientspecific dual compartment breast phantom for validating MRI acquisition and analysis techniques Transactions on Additive Manufacturing Meets Medicine 1

Zhang F, Zhang H, Zhao H, He Z, Shi L, He Y, Ju N, Rong Y and Qiu J 2019 Design and fabrication of a personalized anthropomorphic phantom using 3D printing and tissue equivalent materials Quant Imaging Med Surg 9 94-100

Zhang Y, Boye D, Tanner C, Lomax A J and Knopf A 2012 Respiratory liver motion estimation and its effect on scanned proton beam therapy Physics in medicine and biology 571779

Zhang Y, Huth I, Wegner M, Weber D C and Lomax A J 2016 An evaluation of rescanning technique for liver tumour treatments using a commercial PBS proton therapy system Radiotherapy and Oncology 121 281-7 